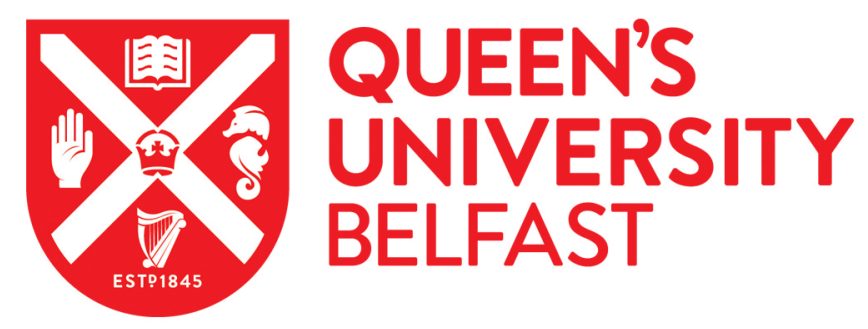

\title{
The frequency and management of seizures during psychological treatment among patients with psychogenic nonepileptic seizures and epilepsy
}

Kemp, S., Graham, C. D., Chan, R., Kitchingman, H., Vickerman, K., \& Reuber, M. (2018). The frequency and management of seizures during psychological treatment among patients with psychogenic nonepileptic seizures and epilepsy. Epilepsia, 59(4), 844-853. https://doi.org/10.1111/epi.14040

Published in:

Epilepsia

Document Version:

Peer reviewed version

Queen's University Belfast - Research Portal:

Link to publication record in Queen's University Belfast Research Portal

Publisher rights

(C) 2018 International League Against Epilepsy.

This work is made available online in accordance with the publisher's policies. Please refer to any applicable terms of use of the publisher.

\section{General rights}

Copyright for the publications made accessible via the Queen's University Belfast Research Portal is retained by the author(s) and / or other copyright owners and it is a condition of accessing these publications that users recognise and abide by the legal requirements associated with these rights.

Take down policy

The Research Portal is Queen's institutional repository that provides access to Queen's research output. Every effort has been made to ensure that content in the Research Portal does not infringe any person's rights, or applicable UK laws. If you discover content in the Research Portal that you believe breaches copyright or violates any law, please contact openaccess@qub.ac.uk. 


\title{
The frequency and management of seizures during psychological treatment
} among patients with psychogenic non-epileptic seizures and epilepsy.

\author{
Steven Kemp [1] ${ }^{\star}$, Christopher D. Graham [1, 2], Rebecca Chan [1], \\ Hayley Kitchingman [1], Kirsty Vickerman [3], Markus Reuber [3].
}

[1] Department of Clinical and Health Psychology, St James's University Hospital, Leeds, UK

[2] Leeds Institute of Health Sciences, University of Leeds, Leeds, UK

[3] Academic Neurology Unit, University of Sheffield, Royal Hallamshire Hospital, Sheffield, UK

${ }^{*}$ Corresponding author

Dr Steven Kemp

Department of Clinical and Health Psychology

St James's University Hospital

Leeds (UK)

LS9 7TF

Fax: 01132064079

Email: stevenkemp@nhs.net

Total word count: 1712

Key words: epileptic seizures, psychogenic non-epileptic seizures, differential diagnosis 
Abstract

Objective: Growing evidence suggests that psychological treatments are effective for improving outcomes in both epilepsy and psychogenic non-epileptic seizures (PNES). However, the risk of in-session seizures may cause concerns about safety and scope of psychological interventions. This study explores the risk of in-session seizures in patients with epilepsy and those with PNES, the timings of seizures during psychological therapy and therapists' responses to seizures.

Methods: Consecutive patients with epilepsy or PNES attending two neurology centres in the United Kingdom for psychological treatment to help with their seizure disorders were studied. Information about seizures during outpatient psychological therapy sessions was gathered using a 12-item pro-forma.

Results: 97 patients with epilepsy and 195 with PNES were captured. One in 32 patients with epilepsy and 1 in 8 with PNES had at least one in-session seizure. A seizure occurred in 1 in 136 treatment sessions provided to patients with epilepsy, and 1 in 36 sessions provided to those with PNES. The risk of in-session seizures was significantly greater in patients with PNES than epilepsy (odds ratio 4.42, 95\% Cl 1.29-15.15). Seizures tended to occur in the first half of treatment programs and individual sessions and only disrupted sessions briefly. Only one patient with PNES required in-patient observation not involving overnight admission.

Significance: In-session seizures do occur, and are much more common in patients with PNES than those with epilepsy. Seizures rarely caused major disruption to psychological treatment, and could almost invariably be managed by the treating therapist without help from additional medical staff. Nonetheless, this research suggests that psychological therapy providers should anticipate the occurrence of insession seizures and have safe management plans in place. The greater frequency of in-session seizures in PNES adds to our understanding of the mechanisms triggering these seizures.

\section{Current word count 290}




\section{Bullet points}

- Seizures do occur in psychological treatment sessions

- In-session seizures are much more common in PNES (1 in 36 sessions) than in epilepsy ( 1 in 136 sessions)

- Seizures are more likely in the first few psychological therapy sessions.

- Most in-session seizures are readily managed by therapists without external medical assistance

- Psychological treatment providers should have seizure management plans in place when treating people with seizure disorders 


\section{Introduction}

Epilepsy is a chronic disorder defined by an increased risk of recurrent, unprovoked epileptic seizures. Psychogenic non-epileptic seizure disorders (PNES) comprise paroxysms superficially resembling those seen in epilepsy but not associated with the abnormal electrical activity in the brain which characterises epilepsy. Most PNES are understood to occur as a manifestation of distress [1] or via attentional and beliefdriven processes ${ }^{[2]}$. Both types of seizure disorders may remit, but become a chronic problem in many cases ${ }^{[3,4]}$.

Neither epilepsy nor PNES disorders are characterised by seizures alone. Cognitive dysfunction and psychiatric comorbidities are more common in patients with both of these seizure disorders than in the general population ${ }^{[5,6,7,8]}$, and the complex and intimate nature of the links between seizures and psychiatric and cognitive morbidity is recognised in the ILAE definition of epilepsy [9]. Indeed, when epileptic seizures or PNES are not fully controlled by treatment, neuropsychological and psychiatric problems have greater adverse effects on patients' quality of life than seizure-related variables ${ }^{[5,6,7,8,10,11]}$. There is accumulating evidence of the effectiveness of psychological therapy in patients with both types of seizure disorders: such treatments can improve health related quality of life (HRQoL) in patients with epilepsy [12], and seizure control, HRQoL, and functioning in those with PNES ${ }^{[13,14]}$. Consequently, in clinical practice a proportion of people with PNES and epilepsy may be referred for psychological therapy.

However, while there is a clear role for psychological treatment in patients with both seizure disorders, recent surveys in the United States, United Kingdom (UK) and globally suggest that even in the case of PNES, where psychological intervention is considered the treatment modality of choice, access to psychological assessment and treatment remains patchy ${ }^{[15,16,17]}$. While this may be the result of a number of factors, one potential barrier to access might involve the extent to which psychology services are equipped to manage the occurrence of seizures. For example, in many places psychological treatment services specialising in the treatment of patients with seizures are not available or accessible and generic services (such as primary care 
or community mental health services) become involved. Less specialised services may lack immediate access to medical support, so the occurrence of seizures (or concerns about managing seizures) could cause significant practical problems.

It is presently not known how often seizures occur in the context of psychological therapy sessions, how they are managed by psychological therapists when they happen and the extent to which they interfere with therapy. Such information can help guide the practice of psychological therapy services that treat people with seizure disorders. Consequently, this study was designed to determine the prevalence of seizures during outpatient psychological therapy sessions. It explores:

i) whether in-session seizures are more likely in PNES or epilepsy; ii) the characteristics of in-session seizures; and, (iii) how seizures are managed by psychological therapists.

\section{Method}

\subsection{Setting}

Data for this study were collected from two clinical neuroscience centres in the UK: The Specialised Neuropsychotherapy Service based within the Department of Neurology of the Sheffield Teaching Hospitals NHS Foundation Trust (centre 1) and the Department of Clinical Neuropsychology at the Leeds Teaching Hospitals NHS Trust (centre 2). Patients were seen by one of eight therapists. Consecutive recruitment was prospective at centre 1 (February 2014 to April 2015) and retrospective at centre 2 (data retrieved from records of patient seen between April 2010 and March 2015). This study was approved as a Service Evaluation by the Clinical Effectiveness Units of the participating hospitals.

\subsection{Patients}

All patients had diagnoses of epilepsy or PNES and had been referred to the participating psychological therapy services for psychological assessment and treatment. All seizure disorders were diagnosed by fully-trained neurologists. Diagnoses were based on all available clinical information (including seizure description, brain imaging and EEG findings, but not always including the video-EEG 
recording of typical seizures). All diagnoses of PNES were sufficiently certain for neurologists to recommend psychological treatment and stop antiepileptic drug treatments (if previously prescribed). Patients with uncertain diagnoses or mixed (epileptic / non-epileptic) seizure disorders were not included. In both centres, patients with epilepsy receive psychological therapy aimed at addressing adjustment problems, depression, anxiety, seizure phobia and posttraumatic symptoms relating to the epilepsy, with less urgency on managing specific emotional triggers which do not generally lead to 'epileptic' seizures. Patients with PNES on the other hand engage in various forms of psychological therapy including integrative, cognitive behavioural (CBT), sensori-motor, Gestalt, psychodynamic and various trauma based paradigms, with an additional initial focus on grounding and stabilisation techniques in centre 1. The therapeutic work focuses on processing trauma and loss, with the ultimate aim of improving emotional processing and psychological self-care.

\subsection{Measures and Procedure}

This study comprised an audit of routinely collected data. Data for this study was collected on a pro-forma (provided as supplementary online information, appendix A). The pro-forma was used to collect information about the nature, precise timing, clinical context, treatment and consequences of in-session seizures. At centre 1 this pro-forma was completed immediately after treatment sessions in which a seizure had been observed by the therapist providing the psychological intervention. At centre 2, the pro-forma was completed retrospectively from the detailed contemporaneous treatment records by two assistant psychologists and one research assistant, working under the supervision of a Consultant Clinical Neuropsychologist. Data about the number of treatment sessions provided to patients with seizures in both centres, the total number of patients seen and treatment sessions provided during the study period were obtained from the participating hospitals' patient administration systems.

\subsection{Data Analysis}

Data were analysed using SPSS (version 17). Between-group differences were analysed using chi-square test. The association between diagnosis and occurrence 
of in-session seizures was analysed using chi-square test. In a logistic regression analysis comparing seizures in patients with epilepsy and PNES, baseline seizure frequency was entered as block 1 and in-session seizure occurrence as block 2 .

\section{Results}

A total of 292 patients with seizures attended psychological therapy at the two centres during the recruitment period, 97 with epilepsy and 195 with PNES.

Descriptive data on all 292 patients is set out in Table 1. Descriptive data on patients who had in-session seizures is set out in Table 2. There was a gender difference between the groups, with more female patients in the PNES group. PNES patients also reported a higher seizure frequency compared to those with epilepsy (i.e. median $=10$ and 4 seizures per month respectively). The median number of psychological treatment sessions attended was 6 for epilepsy patients and 5 for PNES patients.

Insert Table 1: about here

Insert Table 2: about here

The total number of treatment sessions provided to patients with epilepsy during the whole recruitment period at the two centres was 678 . The total number of treatment sessions provided to patients with PNES during the data collection period was 1,356 .

Three patients with epilepsy experienced at least one seizure during psychological therapy. Among these three participants there were seven seizures in total. In comparison, 26 patients with PNES experienced at least one in-session seizure, with 48 in-session seizures in total. This means that one in 32 patients with epilepsy and one in eight with PNES had at least one in-session seizure. One or more seizures occurred in one in 136 treatment sessions provided to patients with epilepsy, and in one in 36 sessions involving patients with PNES. 
Table 3 shows the logistic regression analysis with diagnostic group (epilepsy vs. PNES) as the dependent variable. First, we controlled for seizure frequency, which was not an independent predictor of diagnostic group $(p<.141)$. Secondly, we controlled for gender, which was an independent predictor of diagnostic group $(\mathrm{p}<$ .042). The addition of in-session seizure (present vs. absent) showed this to be an independent predictor $(p<.018)$ with an odds ratio of $4.42(95 \% \mathrm{Cl} 1.29-15.15)$. Therefore patients who had in-session seizures were four times more likely to have PNES than epilepsy.

Insert Table 3: about here

Seizures occurred more commonly in the first half of the psychological treatment programme and of individual treatment sessions than the second. Epileptic seizures tended to be shorter than PNES but three quarters of PNES resolved in less than five minutes. The vast majority of seizures stopped with "just waiting". Therapists arranged temporary observation (without admission overnight) on a neurology ward because of three consecutive PNES in one case. These measures were never considered necessary for any of the observed epileptic seizures, although epileptic seizures prompted therapists more often than PNES to make patients safe or ask a relative or carer to help manage the seizure (see Table 4 for descriptive data of observed seizures).

\section{Discussion}

Our study demonstrates that the occurrence in-session seizures is a feature of psychological therapy sessions with patients with seizure disorders. They are significantly more common in sessions with patients with PNES than those with epilepsy - occurring in 1 in 136 sessions with patients with epilepsy and 1 in 36 sessions involving those with PNES.

This difference between the groups was not explained by the greater seizure frequency reported by patients with PNES, with the group difference remaining 
significant after controlling for this variable. Thus, while our dataset does not allow us to disentangle the exact reasons for the greater frequency of in-session seizures in those with PNES, these observations suggest that aspects of psychological therapy sessions can trigger these seizures. Given that these sessions explore seizure triggers, mood and a person's understanding of their seizures, it may be that PNES are triggered by the activations of seizure representations, by the modulation of mood or the level of arousal ${ }^{[5,18]}$. This interpretation would be consistent with the integrative cognitive model which argues that PNES represent the activation of a seizure scaffold as a dissociative response to internal or external cues in the context of impaired inhibition [18].

Previous studies have demonstrated that about $50 \%$ of patients receiving psychotherapy for PNES become seizure-free with treatment and about $80 \%$ experience an $>50 \%$ reduction in the frequency of their attacks [13]. Our finding that in-session seizures in PNES are less likely to occur in latter psychological therapy sessions resonates with these observations and suggests that patients may become better able to tolerate emotionally challenging situations as their treatment progresses.

Although there is some evidence suggesting that, like PNES, epileptic seizures can also be triggered by emotional states (and controlled by a modulation of such states) [19], our dataset suggests that, generally speaking, psychological therapy is unlikely to comprise such a trigger for this patient group.

The between-group difference in in-session seizure frequency may also be explained by therapists pursuing a different treatment focus in epilepsy compared to PNES. For example, treatments for PNES may have more often focused on improving patients' tolerance of seizure triggers, or on antecedent psychological trauma; whereas psychological therapy for epilepsy may have focused on finding ways to live well with a chronic disease.

Neither epileptic seizures nor PNES were likely to cause a major disruption of psychological therapy. Almost invariably, seizures were successfully managed by the treating therapist without help from physicians or emergency care staff within the normal time frame of the therapy session. None of the seizures observed caused any injuries. Only one patient with PNES (and none with epilepsy) failed to recover 
sufficiently from their seizures within the time allocated for the therapy session to go home.

Despite the fact that seizures were relatively infrequent in our patient cohort, psychological therapy providers should anticipate the occurrence of in-session seizures in the patient groups described here, and to have safe management plans in place. Both epileptic and non-epileptic seizures can cause serious injuries [20, 21]. Although studies exploring the injury risk in these two seizures disorders have failed to identify clear differences in the reported frequency of injuries ${ }^{[22]}$, the injury risk per seizure is likely to be much lower in PNES. In patients with epilepsy, psychological therapy service providers should also consider the risk of status epilepticus, a potentially life-threatening emergency. In our consecutive cohort medical support was only needed once - for a patient with recurrent PNES. Support was not required because of a life-threatening emergency but rather because PNES were recurrent and appeared unresponsive to the seizure control techniques applied by the therapist. The patient was given extra time within the contained environment of a neurology ward to recover from the prolonged seizure. The patient returned to a normal level of consciousness within half an hour of being brought onto the ward. Although medical support was, therefore, rarely called upon, psychological therapists in both centres had access to this support and could arrange further observation of patients. The ability to call for help when needed, and without exposing patients to the risk of traumatisation by the involvement of emergency services [23], may have helped therapists to feel more confident about tackling potentially distressing aspects of therapy.

\section{Limitations}

This study has two salient limitations. First, the retrospective recording of seizures in one of the two centres may have affected the accuracy of some collected data. Second, given that we recorded only a few variables, we were unable to disentangle the reasons why seizures were more frequent in people with PNES. We also had limited information about the timings of seizures within sessions in relation to session content, which might have identified the aspects of the therapeutic interaction serving as seizure triggers. As mentioned previously, another limitation regarding the 
comparisons between the PNES and epilepsy groups is that, given the differing aetiology of the two conditions, the psychological intervention methods utilised by therapists may have differed between these groups. Last but not least, psychiatric comorbidities may have had an effect on the risk of in-session seizures, and psychiatric comorbidity profiles are likely to have differed between the patients with epilepsy and PNES [5], but relevant data was not collected in a structured fashion in all cases and therefore not available for analysis.

\section{Conclusion}

This study showed that the presence of in-session seizures is a feature of working with patients with seizure disorders, and these are much more common in those with PNES compared to those with epilepsy. Although PNES could almost invariably be managed by the psychological therapist without help from physicians or emergency care staff, we recommend that psychological therapy providers should anticipate the occurrence of in-session seizures and to have safe management plans in place. The fact that PNES occur more commonly than epileptic seizures during psychological therapy sessions adds to our understanding of the mechanisms triggering PNES.

\section{$\underline{\text { References }}$}

1) Wiseman $H$, Reuber $M$. New insights into psychogenic nonepileptic seizures 2011-2014. Seizure. 2015;29:69-80.

2) Edwards MJ, Adams RA, Brown $\mathrm{H}$, et al. A Bayesian account of 'hysteria'. Brain. 2012;135:3495-512.

3) Kwan P, Arzimanoglou A, Berg AT, et al. Definition of drug resistant epilepsy: consensus proposal by the ad hoc Task Force of the ILAE Commission on Therapeutic Strategies. Epilepsia. 2010;51:1069-77.

4) Reuber M, Pukrop R, Bauer J, et al. Outcome in psychogenic nonepileptic seizures: 1 to 10-year follow-up in 164 patients. Ann neurol. 2003;53:305-11.

5) Brown RJ, Reuber M. Psychological and psychiatric aspects of psychogenic nonepileptic seizures (PNES): a systematic review. Clin psychol rev. 2016;45:157-82.

6) Mula M. Cognitive dysfunction in patients with epilepsy: focus on clinical variables. Future Neurol. 2015;10:41-8. 
7) Reuber M. Are psychogenic non-epileptic seizures an expression of "neurologic" pathology?". Psychiatric controversies in epilepsy. 2008:153-77.

8) Tellez-Zenteno JF, Patten SB, Jetté N, et al. Psychiatric comorbidity in epilepsy: a population-based analysis. Epilepsia. 2007;48:2336-44.

9) Fisher RS, Acevedo C, Arzimanoglou A, et al. ILAE official report: a practical clinical definition of epilepsy. Epilepsia. 2014;1:55:475-82.

10)Jacoby A, Snape D, Baker GA. Determinants of quality of life in people with epilepsy. Neurol clin. 2009;27:843-63.

11)Jones $B$, Reuber $M$, Norman $P$. Correlates of health-related quality of life in adults with psychogenic nonepileptic seizures: A systematic review. Epilepsia. 2016;57:171-81.

12)Michaelis $R$, Tang $V$, Wagner $\mathrm{JL}$, et al. Psychological treatment for people with epilepsy. The Cochrane Library. 2016. Under review.

13)Carlson P, Perry KN. Psychological interventions for psychogenic non-epileptic seizures: A meta-analysis. Seizure. 2017;45:142-50.

14)LaFrance WC, Reuber M, Goldstein LH. Management of psychogenic nonepileptic seizures. Epilepsia. 2013;54(s1):53-67.

15)Kanemoto K, LaFrance WC, Duncan R, et al. PNES around the world: where we are now and how we can close the diagnosis and treatment gaps. An ILAE PNES Task Force report. Epilepsia Open. 2017;1-10. doi: 10.1002/epi4.12060.

16)LaFrance WC, Rusch MD, Machan JT. What is "treatment as usual" for nonepileptic seizures?. Epilepsy Behav. 2008;12:388-94.

17)Mayor R, Smith PE, Reuber M. Management of patients with nonepileptic attack disorder in the United Kingdom: a survey of health care professionals. Epilepsy Behav. 2011;21:402-6.

18)Brown RJ, Reuber M. Towards an integrative theory of psychogenic non-epileptic seizures (PNES). Clin psychol rev. 2016;47:55-70.

19)Asadi-Pooya AA, Emami M, Emami Y. Ictal injury in psychogenic non-epileptic seizures. Seizure. 2014;23:363-6.

20)Nguyen R, Zenteno JF. Injuries in epilepsy: a review of its prevalence, risk factors, type of injuries and prevention. Neurol int. 2009;1.

21)Peguero $E$, Abou-Khalil $B$, Fakhoury $T$, et al. Self-Injury and Incontinence in Psychogenic Seizures. Epilepsia. 1995;36:586-91. 
22)Worsely $C$, Whitehead K, Kandler R, et al. Illness perceptions of health care workers in relation to epileptic and psychogenic nonepileptic seizures. Epilepsy Behav. 2011;20:668-73. 
Table 1: Descriptive data for all patients: Clinical characteristics and seizure incidence:

\begin{tabular}{llll}
\hline & PNES & Epilepsy & Total \\
\hline $\mathrm{N}$ & & & \\
Age at first session (median years; range) & 195 & 97 & 292 \\
Gender (\% women/men) & $60(18-87)$ & $39(15-63)$ & $40(15-87)$ \\
Learning disabilities (\%) & 3 & $55 / 45$ & $63 / 37$ \\
$\begin{array}{l}\text { Seizure frequency per month (median; range) } \\
\text { Number of sessions (median; range) }\end{array}$ & $10(0-1500)$ & $4(0-600)$ & 4 \\
& $5(1-26)$ & $6(1-21)$ & $6(1-26)$ \\
$\begin{array}{l}\text { Number of patients that had in-session } \\
\text { seizures (\%) }\end{array}$ & $26(13)$ & $3(3)$ & \\
$\begin{array}{l}\text { Number of patients that did not have seizures } \\
\text { (\%) }\end{array}$ & $169(87)$ & $94(97)$ & \\
& & & \\
\hline
\end{tabular}


Table 2: Descriptive data for patients who had in-session seizures: Incidence of in-session seizures:

\begin{tabular}{|c|c|c|c|}
\hline & PNES & Epilepsy & Total \\
\hline \multicolumn{4}{|l|}{$\mathrm{N}$} \\
\hline Number of patients who had seizures & 26 & 3 & 29 \\
\hline Number of seizures captured & 48 & 7 & 55 \\
\hline $\begin{array}{l}\text { Incidence of seizures in these patients } \\
\text { (median; range) }\end{array}$ & $1(1-10)$ & $3(1-3)$ & \\
\hline $\begin{array}{l}\text { Number of patients that had more than one } \\
\text { seizure }(\%)\end{array}$ & $7(27)$ & $2(67)$ & \\
\hline
\end{tabular}


Table 3: Logistic regression:

\begin{tabular}{|c|c|c|c|c|c|c|c|}
\hline \multirow[t]{2}{*}{ Variable } & \multirow[t]{2}{*}{ B } & \multirow[t]{2}{*}{ SE } & \multirow[t]{2}{*}{ Wald test } & \multirow[t]{2}{*}{ Sig } & \multirow[t]{2}{*}{ Odds ratio } & \multicolumn{2}{|c|}{$95 \% \mathrm{Cl}$} \\
\hline & & & & & & Lower & Upper \\
\hline Constant & 0.708 & 0.174 & 16.592 & 0.000 & 2.029 & & \\
\hline Seizure Frequency & 0.002 & 0.002 & 2.162 & 0.141 & 1.002 & 0.999 & 1.006 \\
\hline Gender & -0.528 & 0.260 & 4.132 & 0.042 & 0.590 & 0.355 & 0.981 \\
\hline $\begin{array}{l}\text { Presence/Absence } \\
\text { of Seizure }\end{array}$ & 1.485 & 0.629 & 5.573 & 0.018 & 4.416 & 1.287 & 15.151 \\
\hline
\end{tabular}


Table 4: Clinical characteristics and management of in-session seizures:

PNES Epilepsy

$n=46 \quad n=7$

Place of attack (\% of seizures)

In hospital transport

0

In the waiting area

0

14

In therapy room

Ambulance waiting area

Elsewhere in the hospital

Not known

$17-14$

$17 \quad 86$

$0 \quad 0$

$6 \quad 0$

Timing of seizure in course of treatment (\%)

First quarter

Second quarter

39

0

Third quarter

Last quarter

During the only session attended

90

Timing of attack in session (\%)

Before the session

$15-14$

First quarter of session

2nd quarter

3rd quarter

Last quarter

Immediately after session

Not known

$27 \quad 29$

$21-0$

Duration of attack (\%)

Less than a minute $\quad 31 \quad 57$

1 minute

2 minutes

$4 \quad 0$

3 minutes

4 minutes

5 minutes

10 minutes

15-30 minutes

Description of attack $(\%)^{\star}$

Seizure warning

Absence

Drop attack

Shaking of limbs

Shaking of body

Some consciousness retained

Consciousness lost completely

Some responsiveness during seizure

Completely unresponsive

0

Speech during episode

Crying during/after episode

Patient looked fearful

Incontinent of urine

Incontinent of faeces

Injuries incurred

Single episode

Multiple episodes

Not known

$31 \quad 43$

6

0

$0 \quad 0$

$0-0$

0

$0-0$

$15-0$

$0 \quad 0$

$17 \quad 0$

Possible trigger $(\%)^{*}$

Traumatic reminder $\quad 33 \quad 0$

Anxiety/panic

Other emotion

$10-29$

Sounds

Other

17 
Were seizure prevention techniques employed by

clinician? (\% yes)

Were seizure prevention techniques considered

Types of seizure prevention techniques used (\% of seizures) *

Talking to the patient $\quad 58$

Breathing 19

Grounding

Other somatic techniques

Distraction

Smelling salts

Rose oil or other perfume

Other

33

0

0

0

4

Other actions employed (\% of seizures, several responses possible)*

$\begin{array}{lll}\text { Just waited } & 75 & 100 \\ \text { Patient kept safe } & 33 & 86 \\ \text { Family member/friend asked for help } & 8 & 43 \\ \text { Service protocol for lengthy in-session } & 4 & 0 \\ \text { seizures used } & & \\ \text { Emergency assistance called for } & 0 & 0 \\ \text { Other } & 13 & 0\end{array}$

Consequence of seizure ( $\%$ of seizures) ${ }^{*}$

Able to go home normally

Had to call relative or friend

Went on neurology ward for observation

Admitted with overnight stay

Other

Number of patients requiring help from relatives or emergency services following a seizure

* Several answers possible 
Pro-forma used

Pilot study - Seizures in the therapy context in epilepsy and non-epileptic attack disorder

Sheffield teaching Hospitals and St James University Hospital, Leeds

Front sheet - Therapist to complete one for each patient

Name of Therapist

1 Patient details

1.1 ID no:

1.2 Age:

1.3 Gender M/F

1.4 The patient is diagnosed with: $\quad$ Epilepsy/NEAD/epilepsy and NEAD

1.5 Duration of seizure disorder years

1.6 Is patient on anti-epileptic medication?

Yes/No

1.7 Does the patient have learning difficulties?

Yes/No

1.8 Do you work with the patient through an interpreter? Yes/No 
Pilot study - Seizures in the therapy context in epilepsy and non-epileptic attack disorder

\section{Episode sheet}

To be completed by therapist for each session of therapy in which patient experiences an actual or threatened seizure.

Name of therapist:

Patient ID no.

Age

Session no.

Date

Number of seizures patient has had in the last week

1. Context of the episode

1.1 Was the patient accompanied to session? Yes/No

1.2 Where did the seizure occur?
a) In hospital transport
b) In the waiting area
c) In therapy room
d) Ambulance waiting area
e) Elsewhere in the hospital
f) Not Known

1.3 Who was present at the time? (tick all that apply)
a) Therapist
b) Family or friend
c) Reception staff
d) Other patients in waiting room
e) General public
f) Other (please specify)
g) Not Known

1.4 When did the episode occur?
a) Before the session
b) $1^{\text {st }}$ quarter
c) $2^{\text {nd }}$ quarter
d) $3^{\text {rd }}$ quarter
e) Last quarter
f) Immediately after session

\section{Nature of episode}

2.1 What best describes the episode? Tick any that apply.
a) Seizure warning
b) Absence
c) Drop attack
d) Shaking of limbs
e) Shaking of body 
f) Some consciousness retained

g) Consciousness lost completely

h) Some responsiveness during seizure

i) Completely unresponsive

j) Speech during episode

k) Crying during/after episode

l) Patient looked fearful

$\mathrm{m})$ Incontinent of urine

n) Incontinent of faeces

o) Injuries incurred

p) Single episode/multiple episodes

q) Not known

2.2 Episode trigger

a) Traumatic reminder

b) Anxiety/panic

c) Other emotion

d) Sounds

e) Other (please specify)

f) No trigger apparent

g) Not known

2.3 Duration of total episode

minutes

2.4 Was the episode over by the end of the session?

Yes/No

2.5 What happened afterwards?

a) Able to go home normally

b) Had to call relative or friend

c) Went on neurology ward for observation

d) Admitted with overnight stay

e) Other

\section{Actions taken by therapist}

3.1 Were seizure prevention techniques used

Yes/No

3.1.1 If yes, which techniques were used?
a) Talking to patient
b) Breathing
c) Grounding
d) Other somatic techniques
e) Distraction
f) Smelling salts
g) Rose oil or other perfumes
h) Other

3.1.2. Were the techniques successful?

Yes/No/Partly

3.2 What other action was taken by the therapist? Tick all that apply.

a) Just waited

b) Patient kept safe 
c) Family member/friend asked for help

d) Service protocol for lengthy in-session seizures used

e) Emergency assistance called for

f) Other (please specify) 